\title{
How can nanovectors be used to treat spinal cord injury?
}

\author{
Filippo Rossi*,1 (D), Simonetta Papa², Giuseppe Perale ${ }^{3,4}$ \& Pietro Veglianese ${ }^{2}$ \\ ${ }^{1}$ Department of Chemistry, Materials \& Chemical Engineering 'Giulio Natta', Politecnico di Milano, via Mancinelli 7, 20131 Milan, \\ Italy \\ 2Department of Neuroscience, IRCCS Istituto di Ricerche Farmacologiche 'Mario Negri', via La Masa 19, 20156 Milan, Italy \\ ${ }^{3}$ Biomaterials Laboratory, Institute for Mechanical Engineering \& Materials Technology, University of Applied Sciences and Arts of \\ Southern Switzerland, via Cantonale 2C, Galleria 26928 Manno, Switzerland \\ ${ }^{4}$ Ludwig Boltzmann Institute for Experimental \& Clinical Traumatology, Donaueschingenstrasse 13, 1200 Vienna, Austria \\ *Author for correspondence: filippo.rossi@polimi.it
}

\section{"Polymeric NPs seem to offer the best way to deliver drugs selectively in microglia, astrocytes and neurons, which are the key actors in $\mathrm{SCl} . "$}

First draft submitted: 23 September 2019; Accepted for publication: 25 October 2019; Published online: 19 December 2019

Keywords: colloids $\bullet$ drug delivery $\bullet$ multitherapy $\bullet$ nanoparticles $\bullet$ spinal cord injury

\section{Spinal cord injury}

Spinal cord injury (SCI) is a devastating condition that chronically affects approximately 2.5 million people worldwide and every year, 130,000 new cases are recorded (wingsforlife.com), with high physical, social and economic impacts [1,2]. SCI is the result of two different phases: primary injury and secondary injury. Primary injury as a consequence of a traumatic event, such as motorbike or car accidents, sport traumas or gunshots that cause synthesis of nitric oxide, oxidative stress, microglia activation and local inflammation. However, most of the post traumatic degeneration of the cord is caused by the secondary injury, a multifactorial process with neuronal necrosis, scar and cyst formation, demyelination and disruption of morpho-functional nerve pathways [3,4]. The results are related to sensory and motor dysfunctions below the level of the lesion, with chronic pain syndrome. The common procedure in hospitals is directed to patient stabilization and decompression of the spinal cord, with systemic administration of corticosteroids (methylprednisolone). However, this approach is not always effective, because it is mainly intended to reduce inflammation and pain, to limit further damage and lesion extensions, and this is why the research for effective treatment of SCI is still a big challenge.

In addition, a common conventional treatment strategy is the use of infusion with mini pumps. While the immediate drug efficacy offers some advantages, several problems restrict the applicability of this administration route like limited drug diffusion into the spinal cord, prompt cerebrospinal fluid clearance and problems related to catheter placement and infections.

\section{Can controlled drug-delivery systems improve pharmacological treatments?}

In the last few decades, research has made a lot of progress on the knowledge of the entire pathophysiology of SCI, clarifying that the main reasons for the complexity of the secondary injury are related to the different kinetics of the phenomena involved.

The different stages of the secondary injury can be divided into inflammation, gliosis and neuroregeneration. It is, therefore, obvious that a promising therapy should involve targeting multiple pathological events with a multitherapy, where different compounds are combined to satisfy different medical needs [5]. In addition, due to the other problems associated with SCI, such as brain trauma, the necessity to have a single administration is pivotal in critical patient management. In this direction, controlled drug-delivery systems demonstrated to be very promising. The idea is to avoid under and over dosing of drugs within the body in order to maintain hematic concentration within therapeutic values for a prolonged period of time reducing side effects and the amount of drug needed. This strategy, described in several studies [6] besides clinical improvement presents some limitations like: 
low selectivity; drawbacks consequent to unwanted interactions between different drugs and problems in targeting the proper therapeutic window.

\section{Polymeric nanoparticles for multitarget therapy}

A power tool in controlled drug-delivery systems is surely represented by polymeric nanoparticles (NPs) which, once injected, are able to localize and sustain the delivery of drugs selectively within cells in situ, at the lesion site. Indeed, polymeric NPs with their versatility in size, potential surface and hydrophilic or lipophilic characteristics, offer considerable advantages in drug delivery, increasing the selectivity of drugs and controlling their release over time. NPs can be prepared following different strategies, described in many papers and reviews $[7,8]$.

Their first advantage lies in the possibility of controlling and sustaining the release of drugs over the classical administration routes. Bellamkonda research group [9] demonstrated that methylprednisolone loaded within NPs gave a better outcome with lesion reduction in animal models. Shoichet research group loaded poly(lactic-glycolic) acid NPs with growth factors and proteins, showing that such NPs could also be used to tailor and sustain the release of biomolecules, not only drugs [10-12]. The second advantage is related to the possibility of selectively delivering drugs inside cells in order to treat the three main pathological pathways in SCI: inflammation, gliosis and neurodegeneration. The rationale is to reduce inflammation, acting against M1/A1 phenotypes (neuroprotection) and then support neuroregeneration through the action of M2 and A2 phenotypes. Quick release of anti-inflammatory drugs in the early stage of the lesion is important because microglia are the first cells to respond to the injury, activating and then building the glial scar [13]. Our group has reported that poly-e-caprolactone NPs loaded with minocycline can selectively treat microglia, reducing their inflammation and consequently the secondary injury mechanisms associated [14,15]. These results could not be obtained with drugs simply administered systemically, illustrating the importance of nanotools in drug delivery.

Microglia are not the only players in SCI: astrocytes and neurons play key roles too and must be taken onto consideration in a multitarget therapy. Astrocytes with A1 phenotype exert neurotoxic effects, upregulating many genes associated with the synapse and neuronal degeneration, suggesting that A1 has harmful proinflammatory action [16,17]. In contrast, A2-induced reactive astrocytes exert protective effects by upregulating the expression of neurotrophic factors, that promote neuronal survival and regrowth. Various approaches have been employed to counteract the negative effects of activated astrocytes, blocking scar formation or pharmacologically and genetically reducing upregulation of axon growth inhibitors. However, genetic manipulation is not clinically feasible, because ethical issues remain and it may have side effects, whereas pharmacological treatment could act on unwanted common mechanisms without any selective effect on astrocytes.

Again, polymeric NPs seem very promising. In the past decade a large number of different NP systems have been tested in SCI, but only a few are directed to astrocytes, and none are selective $[18,19]$. Then neuroregeneration should be induced and a promising possibility is represented by the selective treatment of neurons and this represents another big challenge.

The key difference is that while microglia and macrophages in general have vigorous phagocytic activity, with great ability to take up foreign bodies (in this case NPs) this is not the case of neurons. It is, therefore, fundamental to understand how the active mechanisms to pass the neuronal membrane work, so as to design selective NPs. While microglia use clathrin-dependent endocytosis as their principal mechanism in cell uptake, neurons do not have this ability. Some researchers have suggested that neuron uptake could be possible using the ability of apoliprotein (ApoE) to release lipids within the neuron, so lipid-based NPs might be a good idea [20].

In summary, the processes involved in SCI differ widely, with different kinetics, so a platform to work selectively at different times could be a winning strategy. Polymeric NPs seem to offer the best way to deliver drugs selectively in microglia, astrocytes and neurons, which are the key actors in SCI. While good results have been obtained with microglia in vitro and in vivo, taking advantage of their phagocytic activity, this is not true for astrocytes and neurons. Research is now oriented to astrocytes and some interesting results have been obtained but only in vitro, while with neurons there is a wide range of possible prospects. In summary, simultaneous drug delivery to microglia, astrocytes and neurons to build up a combination treatment for SCI using polymeric NPs poses a big challenge, but also a great opportunity for synergy between the effect of single treatments to counteract the secondary injury and rebuild the damaged tissue. 


\section{Author contributions}

F Rossi wrote the first draft of the manuscript. S Papa, G Perale and P Veglianese contributed to the final version of the manuscript. All authors provided critical feedback.

\section{Financial \& competing interests disclosure}

The authors have no relevant affiliations or financial involvement with any organization or entity with a financial interest in or financial conflict with the subject matter or materials discussed in the manuscript. This includes employment, consultancies, honoraria, stock ownership or options, expert testimony, grants or patents received or pending, or royalties.

This manuscript was checked by a native English speaker.

\section{References}

1. Singh A, Tetreault L, Kalsi-Ryan S, Nouri A, Fehlings MG. Global prevalence and incidence of traumatic spinal cord injury. Clin. Epidemiol. 6, 309-331 (2014).

2. Priebe MM, Chiodo AE, Scelza WM, Kirshblum SC, Wuermser LA, Ho CH. Spinal cord injury medicine. 6. Economic and societal issues in spinal cord injury. Arch. Phys. Med. Rehabil. 88, S84-S88 (2007).

3. Bradbury EJ, Burnside ER. Moving beyond the glial scar for spinal cord repair. Nat. Commun. 10, 3879 (2019).

4. Silva NA, Sousa N, Reis RL, Salgado AJ. From basics to clinical: a comprehensive review on spinal cord injury. Prog. Neurobiol. 114, 25-57 (2014).

5. Papa S, Rossi F, Vismara I, Forloni G, Veglianese P. Nanovector-mediated drug delivery in spinal cord injury: a multitarget approach. ACS Chem. Neurosci. 10, 1173-1182 (2019).

6. Ziemba AM, Gilbert RJ. Biomaterials for local, controlled drug delivery to the injured spinal cord. Front. Pharmacol. 10, 245 (2017).

7. Behzadi S, Serpooshan V, Tao W et al. Cellular uptake of nanoparticles: journey inside the cell. Chem. Soc. Rev. 46, 4218-4244 (2017).

8. Calzoni E, Cesaretti A, Polchi A, Di Michele A, Tancini B, Emiliani C. Biocompatible polymer nanoparticles for drug delivery applications in cancer and neurodegenerative disorder therapies. J. Funct. Biomater. 10, E4 (2019).

9. Kim Y, Caldwell JM, Bellamkonda R. Nanoparticle-mediated local delivery of methylprednisolone after 1068 spinal cord injury. Biomaterials 30, 2582-2590 (2009).

10. Kang CE, Baumann MD, Tator CH, Shoichet MS. Localized and sustained delivery of fibroblast growth factor-2 from a nanoparticle-hydrogel composite for treatment of spinal cord injury. Cells Tissues Organs 197, 55-63 (2013).

11. Pakulska MM, Donaghue IE, Obermeyer JM et al. Encapsulation-free controlled release: electrostatic adsorption eliminates the need for protein encapsulation in PLGA nanoparticles. Sci. Adv. 2, e160051 (2016).

12. Donaghue IE, Tator CH, Shoichet MS. Sustained delivery of bioactive neurotrophin-3 to the injured spinal cord. Biomater. Sci. 3, 65-72 (2015).

13. Saxena T, Loomis KH, Pai SB et al. Nanocarrier-mediated inhibition of macrophage migration inhibitory factor attenuates secondary injury after spinal cord injury. ACS Nano 9, 1492-1505 (2015).

14. Papa S, Rossi F, Ferrari R et al. Selective nanovector mediated treatment of activated proinflammatory microglia/macrophages in spinal cord injury. ACS Nano 7, 9881-9895 (2013).

15. Papa S, Caron I, Erba E et al. Early modulation of pro-inflammatory microglia by minocycline loaded nanoparticles confers long lasting protection after spinal cord injury. Biomaterials 75, 13-24 (2016).

16. Liddelow SA, Barres BA. Reactive astrocytes: production, function, and therapeutic potential. Immunity 46, 957-967 (2017).

17. Liddelow SA, Guttenplan KA, Clarke LE et al. Neurotoxic reactive astrocytes are induced by activated microglia. Nature 54, 481-487 (2017).

18. Cerqueira SR, Silva NA, Oliveira JM et al. Multifunctionalized CMCht/PAMAM dendrimer nanoparticles modulate the cellular uptake by astrocytes and oligodendrocytes in primary cultures of glial cells. Macromol. Biosci. 16, 299 (2016).

19. Li X, Kozielski K, Cheng YH et al. Nanoparticle-mediated conversion of primary human astrocytes into neurons and oligodendrocytes. Biomater. Sci. 4, 1100-1112 (2016).

20. Zhang F, Lin YA, Kannan S, Kannan RM. Targeting specific cells in the brain with nanomedicines for CNS 795 therapies. J. Control. Rel. 240, 212-226 (2016). 